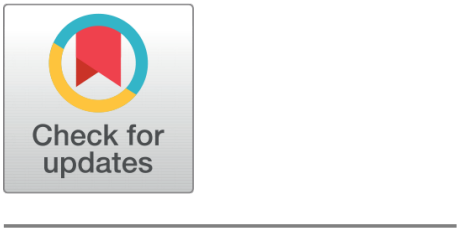

G OPEN ACCESS

Received: 25-04-2020

Accepted: 11-05-2020

Published: 08-06-2020

Editor: Dr. Natarajan Gajendran

Citation: Arohan R, Sharma RK (2020) Periodic orbits in the planar restricted photo-gravitational problem when the smaller primary is an oblate spheroid. Indian Journal of Science and Technology 13(16): 1630-1640. https://doi.org/ 10.17485/IJST/v13i16.401

* Corresponding author. Ritesh Arohan

Department of Aerospace Engineering, Karunya Institute of Technology and Sciences, Coimbatore, 641114, Tamil Nadu, India

ritesharohan8@gmail.com

Funding: None

Competing Interests: None

Copyright: (c) 2020 Arohan, Sharma. This is an open access article distributed under the terms of the Creative Commons Attribution License, which permits unrestricted use, distribution, and reproduction in any medium, provided the original author and source are credited.

Published By Indian Society for Education and Environment (iSee)

\section{Periodic orbits in the planar restricted photo-gravitational problem when the smaller primary is an oblate spheroid}

\author{
Ritesh Arohan $^{1 *}$, Ram Krishan Sharma1 \\ 1 Department of Aerospace Engineering, Karunya Institute of Technology and Sciences, \\ Coimbatore, 641114, Tamil Nadu, India
}

\section{Abstract}

Background/Objectives: This study deals wit $h$ the stationary solutions of the planar circular restricted three-body problem when the more massive primary is a source of radiation and the smaller primary is an oblate spheroid with its equatorial plane coincident with the plane of motion. The objective is to study the location of the Lagrangian points and to find the values of critical mass. Also, to study the periodic orbits around the Lagrangian points. Methods: A new mean motion expression by including the secular perturbation due to oblateness utilized by ${ }^{(1,2)}$ is used in the present studies. The characteristic roots are obtained by linearizing the equation of the motion around the Lagrangian points. Findings: The critical mass parameter $\mu \mathrm{crit}^{(3,4)}$, which decreases radiation force, whereas it increases with oblateness when we consider the value of new mean motion. Through special choice of initial conditions, retrograde elliptical periodic orbits exist for the case $\mu=\mu$ crit, whose eccentricity increases with oblateness and decreases with radiation force for non-zero oblateness, although there is slight variation in $\mathrm{L}_{2}$ location.

Keywords: Restricted three body problem; Lagrangian points; Eccentricity; Oblateness; Critical mass; Radiation force; Mean motion.

\section{Introduction}

The three-body problem, in general, is about the study of three massive bodies $(\mathrm{m} 1$, $\mathrm{m} 2, \mathrm{~m} 3$ ) in space which affect one another by their gravitational forces. In the restricted three-body problem, one of these three bodies are considered to be very small and that it has a very less mass which does not affect the motion of the other two bodies called primaries with masses $\mathrm{m} 1$ and $\mathrm{m} 2$, but it is affected by their mutual forces. The circular restricted three-body problem (CR3BP) studies the motion of a body with a negligible mass under the influence of two bigger bodies, called the primaries, which revolve around their center of mass in ' $n$ ' circular orbit. To make the problem more applicable to real cases, perturbations are also included. Here, time $(t)$ is the independent variable on which the other variables depend. When the restricted three- body problem is solved, it has been observed that there are 5 points on the plane of the primaries where the gravitational forces of the primaries nullify each other and there is no gravitational 
pull for any object which is placed in these points. These points are called 'Lagrangian Points', 'Libration Points' or 'Equilibrium Points' (L1 to L5). Among these 5 points, three are collinear (L1, L2, L3) which lie in the line connecting the two primaries. The other two (L4 and L5) form equilateral triangles with the two primaries above and below the line connecting the primaries.

Since the Sun - the source of radiation - is almost spherical, and some of the planets like Saturn and Jupiter whose equatorial and polar radii are approximately 60400,54600 and $71400,67000 \mathrm{~km}$, respectively, and sufficiently oblate, hence a modification is considered that the smaller primary (planet) is an oblate spheroid. The more massive primary, the Sun, is a source of radiation. It is considered that the equatorial plane of the smaller primary is coincident with the plane of motion and only the planar case has been studied.

In this paper, case considered when the gravitation prevails. As the solar radiation pressure force $F_{p}$ changes with the distance by the same law as the gravitational attraction force $F_{g}$ and acts opposite to it. Thus, the Sun's resultant force acting on the particle is

$F=F_{g}-F_{p}=F_{g}\left(1-\frac{F_{p}}{F_{g}}\right)=q F_{g}$,

Where $q=1-F_{p} / F_{g}$ is the mass reduction factor constant for a given particle. It can be expressed in terms of particle radius (a), density $(\delta)$, and the solar radiation pressure efficiency factor k (in CGS units) as

$q=1-\left(\frac{5.6 \times 10^{-5}}{a \delta} k\right)$

Many of the celestial bodies do not have an exact sphere shape. Instead, they have an oblate spheroid shape. An oblate spheroid is obtained by rotating an ellipse about its minor axis i.e., the equatorial radius becomes longer than the polar radius. This oblateness of the planets render a change to the mean motion of orbit of the primaries, because of the variation of gravitation. The effect of oblateness is quantified by the term $J 2$, which varies for each planet. Oblateness $\left(A_{2}\right)=\frac{\left(A E^{2}-A P^{2}\right)}{5 R^{2}} ; \mathrm{AE}, \mathrm{AP}$ being the equatorial and polar radii of the smaller primary, while $\mathrm{R}$ is the distance between the primaries.

In ${ }^{(5)}$ the authors study the periodic orbits numerically for fixed values of the mass parameter and oblateness coefficient of the smaller primary and by changing the radiation pressure and the energy constant. However, the value of oblateness coefficient was taken from ${ }^{(3)}$. In ${ }^{(6)}$ in the frame work of the perturbed photo-gravitational restricted three-body problem, the first order exterior resonant orbits and the first, third and fifth order interior resonant periodic orders were analysed. The location, eccentricity and periodic of the first order exterior and interior resonant orbits are investigated in the unperturbed and perturbed cases for a specific value of Jacobi constant. $\operatorname{In}^{(7)}$ a version of the relativistic restricted three-body problem which includes the effects of oblateness of the secondary and radiation of the primary was considered to determine the positions and analyse the stability of the triangular points. In ${ }^{(8)}$ interior resonance periodic orbits around the Sun in the Sun-Jupiter photogravitational restricted three-body problem by including the oblateness of Jupiter was studied using the method of Poincaré surface of section. In ${ }^{(9)}$ a passive micron size particle in the field of radiating binary stellar system in the framework of circular restricted three body problem influenced from radial radiation pressure and Poynting-Robertson drag (PR drag) on the equilibrium points and their stability in the binary stellar systems RW-Monocerotis and Krüger-60 was studied. In ${ }^{(10)}$ the motion in the vicinity of triangular equilibrium points of the circular restricted three-body problem of a passively gravitating dust particle in the gravitational field for the binaries system (Kruger 60 and Achird) is investigated. The two bodies of the binary are both oblate radiating stars possessing P-R drag.

We have calculated the value of the critical mass parameter, $\mu_{\text {crit }}$. It is found to decrease with radiation force of the more massive primary and increase with oblateness of the smaller primary.

\section{Equation of Motion}

Following the terminology and notation of ${ }^{(11)}$ and the unit of mass is considered equivalent to the sum of the primary masses, the unit of length is equivalent to their separation and the unit of time is such that the Gaussian constant of gravitation is unity. Similar to as in ${ }^{(3,6)}$, the equation of motion in the dimensionless barycentric-synodic coordinate system (x, y) are $x-2 n y=\frac{d \Omega}{d x}$ (1)

$y+2 n x=\frac{d \Omega}{d y}$

where the force function equation is given by,

$$
\Omega=\frac{n^{2}}{2}\left((1-\mu) r_{1}^{2}+\mu r_{2}^{2}\right]+\frac{q(1-\mu)}{r_{1}}+\frac{\mu}{r_{2}}+\frac{\mu A_{2}}{2 r_{2}^{3}}
$$

where $r_{1}$ and $r_{2}$ are the magnitudes of the position of the spacecraft or satellite from the massive and smaller primary, respectively; $q$ is the mass reduction factor of the massive primary and $A_{2}$ is the oblateness coefficient of the smaller primary. The magnitudes of the position of the satellite or spacecraft are given as 
$r_{1}^{2}=(x-\mu)^{2}+y^{2}, r_{2}^{2}=(x+1-\mu)^{2}+y^{2}(3)$

The Jacobian integral of Equations (1) and (2) is

$x^{2}+y^{2}=2 \Omega-c ;(4)$

$C$ is the Jacobian constant.

The curves of zero-velocity are given by

$2 \Omega(x, y)=c$;

The curves are symmetric with respect to the $\mathrm{x}$-axis, since $\Omega(x, y)=\Omega(x, \pm y)$. The singularities of the manifold of the states of motion are located at those points of the curves of zero-velocity, where

$$
\begin{aligned}
& \frac{d \Omega}{d x}=0=\frac{d \Omega}{d y}, \\
& \text { i.e., } \\
& n^{2} x-\frac{q(1-\mu)(x-\mu)}{r_{1}^{3}}-\frac{\mu(x+1-\mu)}{r_{2}^{3}}-\frac{3 \mu A_{2}(x+1-\mu)}{2 r_{2}^{5}}=0,(5) \\
& y\left(n^{2}-\frac{q(1-\mu)}{r_{1}^{3}}-\frac{\mu}{r_{2}^{3}}-\frac{3 A_{2} \mu}{2 r_{2}^{5}}\right]=0
\end{aligned}
$$

\subsection{Mean Motion}

In this study, we have derived mean motion expression including the secular perturbation effects. From ${ }^{(12,13)}$ for planar motion (inclination $\mathrm{i}=0$ ).

$$
\frac{d M_{s}}{d t}=n\left(1+\frac{3 J_{2}}{2 a^{2}\left(1-e^{2}\right)^{\frac{3}{2}}}\right], \frac{d w_{s}}{d t}=n\left(\frac{3 J_{2}}{a^{2}\left(1-e^{2}\right)^{2}}\right], \frac{d \Omega_{s}}{d t}=n\left(\frac{-3 J_{2}}{2 a^{2}\left(1-e^{2}\right)^{2}}\right]
$$

Using the relationship, $n d t=(1-e \cos E) d E$, in the above equation and averaging over one revolution,

$$
\begin{aligned}
& \frac{1}{2 \pi} \int_{0}^{2 \pi} d M_{s}=\frac{1}{2 \pi} \int_{0}^{2 \pi}\left(1+\frac{3 J_{2}}{2 a^{2}\left(1-e^{2}\right)^{\frac{3}{2}}}\right](1-e \cos E) d E, \\
& \frac{1}{2 \pi} \int_{0}^{2 \pi} d \omega_{s}=\frac{1}{2 \pi} \int_{0}^{2 \pi}\left(\frac{3 J_{2}}{a^{2}\left(1-e^{2}\right)^{2}}\right](1-e \cos E) d E, \\
& \frac{1}{2 \pi} \int_{0}^{2 \pi} d \Omega_{s}=\frac{1}{2 \pi} \int_{0}^{2 \pi}\left(\frac{-3 J_{2}}{2 a^{2}\left(1-e^{2}\right)^{2}}\right](1-e \cos E) d E
\end{aligned}
$$

We get

$$
\begin{aligned}
& n=1+\frac{3 J_{2}}{2 a^{2}\left(1-e^{2}\right)^{\frac{3}{2}}}, \\
& \triangle \omega_{s}=\frac{3 J_{2}}{a^{2}\left(1-e^{2}\right)^{2}}, \\
& \triangle \Omega_{s}=\frac{-3 J_{2}}{2 a^{2}\left(1-e^{2}\right)^{2}},
\end{aligned}
$$

The mean motion including the precession effect due to oblateness is calculated as: $\widetilde{n}=\bar{n}+\triangle \omega_{s}+\triangle \Omega_{s}=1+\frac{3 A_{2} R^{2}}{2 a^{2}\left(\left(1-e^{2}\right) R e\right]^{2}}\left(1+\sqrt{1-e^{2}}\right)$

Since our study is based on CR3BP, substituting the value of eccentricity $(\mathrm{e})=0$ in the above equation,

or, $n=1+\frac{3 A_{2} R^{2}}{a^{2} R e^{2}}$ where, $\frac{R^{2}}{a^{2} R e^{2}}=K$

In dimensionless unit

$n=1+3 A_{2}$,

or $n^{2}=1+6 A_{2}$ (taking only first-order terms in $A_{2}$ ). (7)

\section{Equilibrium points location}

When we take $\mathrm{y}=0$, equation (5) determines the location of the collinear points $\mathrm{L}_{1}\left(\mathrm{x}_{1}, 0\right), \mathrm{L}_{2}\left(\mathrm{x}_{2}, 0\right), \mathrm{L}_{3}\left(\mathrm{x}_{3}, 0\right)$ where

$x_{1}=\mu-1-\xi, x_{2}=\mu-1+\xi, x_{3}=\mu+\xi$

$\xi_{1}, \xi_{2}, \xi_{3}$ satisfying the seventh-degree polynomials:

$$
\begin{aligned}
& \left((12 A+2) \xi^{7}+((-12 A-2) \mu+36 A+6) \xi^{6}+((-24 A-4) \mu+36 A+6) \xi^{5}+((2 q-12 A-4) \mu-2 q+12 A+2) \xi^{4}-4 \mu \xi^{3}\right. \\
& \left.+(-3 A-2) \mu \xi^{2}-6 A \mu \xi-3 A \mu\right) /\left(2 \xi^{6}+4 \xi^{5}+2 \xi^{4}\right)=0 \\
& \left(\left((12 A+2) \alpha^{6}+(-24 A-4) \alpha^{5}+(12 A-2 q) \alpha^{4}+4 \alpha^{3}+(-3 A-2) \alpha^{2}+6 A \alpha-3 A\right) \mu+(12 A+2) \mu\right. \\
& \left.+(12 A+2) \alpha^{7}+(-36 A-6) \alpha^{6}+(36 A+6) \alpha^{5}+(2 q-12 A-2) \alpha^{4}\right) /\left(2 \alpha^{6}-4 \alpha^{5}+2 \alpha^{4}\right)=0
\end{aligned}
$$


$\left(\left((12 A+2) \beta^{6}+(48 A+8) \beta^{5}+(2 q+72 A+10) \beta^{4}+(8 q+48 A+4) \beta^{3}+(12 q+9 A) \beta^{2}+8 q \beta+2 q\right) \mu+(12 A+2) \beta^{7}+(48 A+8) \beta^{6}\right.$ $\left.+(-2 q+48 A+8) \beta^{4}+(-8 q+12 A+2) \beta^{3}-12 q \beta^{2}-8 q \beta-2 q\right) /\left(2 \beta^{6}+8 \beta^{5}+12 \beta^{4}+8 \beta^{3}+2 \beta^{2}\right)=0$

where, $\xi_{1}=\xi, \xi_{2}=\alpha, \xi_{3}=\beta$

Solving the equations (5) and (6), where $y \neq 0$ we get

$r_{1}^{3}=q / n^{2}, r_{2}=1(8)$

By the help of equation (7), we can locate the other two points $L_{4} \& L_{5}$. These points forming isosceles triangles with the primaries are called as triangular points. And $r_{1} \leq 1$.

\section{Stability of the libration points}

Replacing $x=a+\xi, y=b+\eta$ in the equations of motion (1) for studying the motion near any of the equilibrium points $\mathrm{L}(\mathrm{a}, \mathrm{b})$, we get the first variational equations as :

$\xi^{\prime \prime}-2 n \eta^{\prime}=\Omega_{x x}(a, b) \xi+\Omega_{x y}(a, b) \eta,(9)$

$\eta "+2 n \xi^{\prime}=\Omega_{x y}(a, b) \xi+\Omega_{y y}(a, b) \eta$

The Characteristic equation of Equations (9) is

$$
\lambda^{4}+\left(4 n^{2}-\Omega_{x x}-\Omega_{y y}\right) \lambda^{2}+\Omega_{x x} \Omega_{y y}-\Omega_{x y}^{2}=0
$$

\subsection{Stability of the collinear points}

At the collinear points, we get

$$
\begin{aligned}
& \Omega_{x x}=n^{2}+\frac{2 q(1-\mu)}{r_{1}^{3}}+\frac{2 \mu}{r_{2}^{3}}+\frac{6 \mu A_{2}}{r_{2}^{5}}>0, \\
& \Omega_{x y}=0, \\
& \Omega_{y y}=n^{2}-\frac{q(1-\mu)}{r_{1}^{2}}-\frac{\mu}{r_{2}^{3}}-\frac{3 A_{2} \mu}{2 r_{2}^{5}} .
\end{aligned}
$$

With some calculation, it can be proved that $\Omega_{y y}<0$ at $L_{1,2,3}$. Consequently,

$\Omega_{x x} \Omega_{y y}-\Omega_{x y}^{2}<0$

We can note that the roots $\lambda_{i}(i=1,2,3,4)$ of the Characteristic Equation (10) are

$\lambda_{1,2}= \pm\left(-\beta_{1}+\left(\beta_{1}^{2}+\beta_{2}^{2}\right)^{\frac{1}{2}}\right]^{\frac{1}{2}}= \pm \lambda$,

$\lambda_{3,4}= \pm\left(-\beta_{1}-\left(\beta_{1}^{2}+\beta_{2}^{2}\right)^{\frac{1}{2}}\right]^{\frac{1}{2}}= \pm i s$,

where

$\beta_{1}=2 n^{2}-\left(\Omega_{x x}+\Omega_{y y}\right) / 2$,

$\beta_{2}^{2}=-\Omega_{x x} \Omega_{y y}>0$.

The General solution of Equations (9) can be written as

$$
\begin{aligned}
& \xi=\sum \underset{i=1}{4} \alpha_{i} e^{\lambda_{i} t}, \eta=\sum \gamma_{i=1}^{4} e^{\lambda_{i} t},(11) \\
& \text { And } \\
& \left(\lambda_{i}^{2}-\Omega_{x x}\right) \alpha_{i}=\left(2 n \lambda_{i}+\Omega_{x y}\right) \gamma_{i} .
\end{aligned}
$$

It is noted that $\lambda_{1,2}$ are real and $\lambda_{3,4}$ are pure imaginary. Hence, in general case the collinear equilibria are unstable. However, according to ${ }^{(4)}$, we can choose the initial conditions $\left(\xi_{0}, \eta_{0}\right)$ such that $\alpha_{1,2}=0$ and then Equations (11) represent an ellipse whose eccentricity is given by

$\sqrt{1-\beta_{3}^{-2}}$

respectively, where

$\beta_{3}=\left(s^{2}+\Omega_{x x}\right) / 2 n s$

Considering a theoretical problem. Where, $\mu=0.1,0.2,0.3 ; \varepsilon=0,0.01$ and $\mathrm{A}_{2}$ (oblateness) $=0,0.01$.

Properties and nature of $L_{1}$.

We can see that, eccentricity increases with oblateness and slightly increases with radiation pressure. And orbit is elliptical. $P$ roperties and nature of $L_{2}$.

We can see that, eccentricity increases with oblateness and eccentricity decreases with radiation force. And orbit is elliptical. 
Table 1. Locationof $\mathrm{L}_{1}$ and eccentricity of conditional periodic orbits around $\mathrm{L}_{1}$.

\begin{tabular}{llllll}
\hline$\mu$ & $\varepsilon$ & $A_{2}$ & $\xi$ & $\mathrm{x}_{1}$ & \multirow{2}{*}{$\begin{array}{l}\text { Eccentricity (e) } \\
0.92543667\end{array}$} \\
\cline { 1 - 4 } 0.1 & 0 & 0 & 0.35969983 & -1.25969983 & 0.92572111 \\
0.1 & 0.01 & 0 & 0.35889138 & -1.25889138 & 0.94471622 \\
0.2 & 0.01 & 0.01 & 0.36100212 & -1.26100212 & 0.91600058 \\
0.2 & 0 & 0 & 0.47104869 & -1.27104869 & 0.91619536 \\
0.2 & 0.01 & 0 & 0.47035553 & -1.27035553 & 0.93117171 \\
0.3 & 0.01 & 0.01 & 0.46785205 & -1.26785205 & 0.90787718 \\
0.3 & 0 & 0 & 0.55673469 & -1.25673469 & 0.90802208 \\
0.3 & 0.01 & 0 & 0.55613915 & -1.25613915 & 0.92163148 \\
\hline
\end{tabular}

Table 2. Location of $\mathrm{L}_{2}$ and eccentricity of conditional periodic orbits around $\mathrm{L}_{2}$.

\begin{tabular}{llllll}
\hline$\mu$ & $\varepsilon$ & $A_{2}$ & $\xi$ & $\mathrm{x}_{2}$ & Eccentricity $(\mathrm{e})$ \\
\hline 0.1 & 0 & 0 & 0.29096488 & -0.60903511 & 0.96842307 \\
0.1 & 0.01 & 0 & 0.29223645 & -0.60776354 & 0.96813137 \\
0.1 & 0.01 & 0.01 & 0.30720465 & -0.59279534 & 0.97304460 \\
0.2 & 0 & 0 & 0.36192404 & -0.43807595 & 0.97132709 \\
0.2 & 0.01 & 0 & 0.36319101 & -0.43680898 & 0.97111656 \\
0.2 & 0.01 & 0.01 & 0.37481534 & -0.42518465 & 0.97382094 \\
0.3 & 0 & 0 & 0.41387021 & -0.28612978 & 0.97276616 \\
0.3 & 0.01 & 0 & 0.41511790 & -0.28488209 & 0.97092712 \\
0.3 & 0.01 & 0.01 & 0.42467365 & -0.27532634 & 0.97438134 \\
\hline
\end{tabular}

Table 3. Location of $\mathrm{L}_{3}$ and eccentricity of conditional periodic orbits around $\mathrm{L}_{3}$.

\begin{tabular}{llllll}
\hline$\mu$ & $\varepsilon$ & $A_{2}$ & $\xi$ & $\mathrm{x}_{3}$ & Eccentricity (e) \\
\hline 0.1 & 0 & 0 & 0.94160890 & 1.04160890 & 0.86835862 \\
0.1 & 0.01 & 0 & 0.93840960 & 1.03840960 & 0.86836855 \\
0.1 & 0.01 & 0.01 & 0.91962433 & 1.01962433 & 0.87972477 \\
0.2 & 0 & 0 & 0.88283946 & 1.08283946 & 0.87750511 \\
0.2 & 0.01 & 0 & 0.87979735 & 1.07979735 & 0.87333010 \\
0.2 & 0.01 & 0.01 & 0.86147227 & 1.06147227 & 0.88140032 \\
0.3 & 0 & 0 & 0.82320559 & 1.12320559 & 0.87932469 \\
0.3 & 0.01 & 0 & 0.82033371 & 1.12033371 & 0.87936718 \\
0.3 & 0.01 & 0.01 & 0.80254908 & 1.10254908 & 0.88560716 \\
\hline
\end{tabular}

Properties and nature of $L_{3}$.

We see that, eccentricity increases with oblateness, and eccentricity increases slightly with radiation force. And orbit is elliptical.

Considering some real examples from our solar system

Properties and Nature of $L_{1}$.

Properties and nature of $L_{2}$.

Properties and nature of $L_{3}$.

Periodic orbits around the Sun in Sun-Jupiter system for $\mathrm{L}_{1}$ is generated by MATLAB for the mass ratio of $\mu=0.00095333$ and solar radiation pressure $\mathrm{q}=1$ and 0.99 , as shown in [ Figure 1 \& Figure 2].

Periodic orbits around the Sun in Sun-Jupiter system for $\mathrm{L}_{2}$ is generated by MATLAB for the mass ratio of $\mu=0.00095333$ and solar radiation pressure $\mathrm{q}=1$ and 0.99 , as shown in [ Figure 3 \& Figure 4 ].

Periodic orbits around the Sun in Sun-Jupiter system for $\mathrm{L}_{3}$ is generated by MATLAB for the mass ratio of $\mu=0.00095333$ and solar radiation pressure $\mathrm{q}=1$ and 0.99 , as shown in [ Figure 5 \& Figure 6]. 
Table 4. Location of $\mathrm{L}_{1}$ and eccentricity of conditional periodic orbits around $\mathrm{L}_{1}$ for Sun-planet systems.

\begin{tabular}{|c|c|c|c|c|c|}
\hline System & $\mu$ & $A_{2}$ & $\varepsilon$ & $\mathrm{x}_{1}$ & Eccentricity(e) \\
\hline Sun-Mars & $3.2127^{\star} 10^{-7}$ & $5211.14188981^{\star} 10^{-16}$ & 00.0010 .01 & $\begin{array}{r}-1.00475597 \\
-1.00464773 \\
-1.00386702\end{array}$ & $\begin{array}{l}0.94985821 \\
0.92214540 \\
0.96819065\end{array}$ \\
\hline Sun-Jupiter & $9.5333^{\star} 10^{-4}$ & $211.71783905^{\star} 10^{-12}$ & 00.0010 .01 & $\begin{array}{r}-1.02506012 \\
-1.02495138 \\
-1.02401151\end{array}$ & $\begin{array}{l}0.99599793 \\
0.99604663 \\
0.99645211\end{array}$ \\
\hline Sun-Saturn & $2.85639^{\star} 10^{-4}$ & $65.276555555^{\star} 10^{-12}$ & 00.0010 .01 & $\begin{array}{l}-1.04606463 \\
-1.04595722 \\
-1.04501136\end{array}$ & $\begin{array}{l}0.94712293 \\
0.94736490 \\
0.94949755\end{array}$ \\
\hline Sun-Uranus & $4.36431^{\star} 10^{-5}$ & $6713.99789137^{\star} 10^{-16}$ & 00.0010 .01 & $\begin{array}{r}-1.02596188 \\
-1.02585315 \\
-1.02491275\end{array}$ & $\begin{array}{l}0.94255895 \\
0.94300822 \\
0.94691164\end{array}$ \\
\hline Sun-Neptune & $5.14805^{\star} 10^{-5}$ & $2037.88920652^{\star} 10^{-16}$ & 00.0010 .01 & $\begin{array}{r}-1.04117099 \\
-1.04094671 \\
-1.03897779\end{array}$ & $\begin{array}{l}0.89538651 \\
0.89592653 \\
0.90098599\end{array}$ \\
\hline
\end{tabular}

Table 5. Location of $\mathrm{L}_{2}$ and eccentricity of conditional periodic orbits around $\mathrm{L}_{2}$ for Sun-planet systems.

\begin{tabular}{|c|c|c|c|c|c|}
\hline System & $\mu$ & $A_{2}$ & $\varepsilon$ & $\mathrm{x}_{2}$ & Eccentricity $(e)$ \\
\hline Sun-Mars & $3.2127^{\star} 10^{-7}$ & $5211.14188981^{\star} 10^{-16}$ & 00.0010 .01 & $\begin{array}{l}-0.99525841 \\
-0.99514427 \\
-0.99384034\end{array}$ & $\begin{array}{l}0.95049170 \\
0.94809550 \\
0.92227849\end{array}$ \\
\hline Sun-Jupiter & $9.5333^{\star} 10^{-4}$ & $211.71783905^{\star} 10^{-12}$ & 00.0010 .01 & $\begin{array}{l}-0.93237854 \\
-0.93226238 \\
-0.93119681\end{array}$ & $\begin{array}{l}0.95468743 \\
0.95452551 \\
0.95303883\end{array}$ \\
\hline Sun-Saturn & $2.85639^{\star} 10^{-4}$ & $65.276555555^{\star} 10^{-12}$ & 00.0010 .01 & $\begin{array}{l}-0.95475377 \\
-0.95463906 \\
-0.95357825\end{array}$ & $\begin{array}{l}0.95320893 \\
0.95296564 \\
0.95071447\end{array}$ \\
\hline Sun-Uranus & $4.36431^{\star} 10^{-5}$ & $6713.99789137^{\star} 10^{-16}$ & 00.0010 .01 & $\begin{array}{l}-0.97574465 \\
-0.97563121 \\
-0.97455947\end{array}$ & $\begin{array}{l}0.95180148 \\
0.95134427 \\
0.94702807\end{array}$ \\
\hline Sun-Neptune & $5.14805^{\star} 10^{-5}$ & $2037.88920652^{\star} 10^{-16}$ & 00.0010 .01 & $\begin{array}{l}-0.97437855 \\
-0.97426504 \\
-0.97319526\end{array}$ & $\begin{array}{l}0.95189331 \\
0.95146075 \\
0.95327614\end{array}$ \\
\hline
\end{tabular}

Table 6. Location of $\mathrm{L}_{3}$ and eccentricity of conditional periodic orbits around $\mathrm{L}_{3}$ for Sun-planet systems.

\begin{tabular}{|c|c|c|c|c|c|}
\hline System & $\mu$ & $A_{2}$ & $\varepsilon$ & $\mathrm{x}_{3}$ & Eccentricity(e) \\
\hline Sun-Mars & $3.2127^{\star} 10^{-7}$ & $5211.14188981^{\star} 10^{-16}$ & 00.0010 .01 & $\begin{array}{l}1.00000013 \\
0.99966668 \\
0.99665562\end{array}$ & $\begin{array}{l}0.86602540 \\
0.86602540 \\
0.86602512\end{array}$ \\
\hline Sun-Jupiter & $9.5333^{\star} 10^{-4}$ & $211.71783905^{\star} 10^{-12}$ & 00.0010 .01 & $\begin{array}{l}1.00039722 \\
1.00006390 \\
0.99705404\end{array}$ & $\begin{array}{l}0.86602570 \\
0.86602570 \\
0.86602570\end{array}$ \\
\hline Sun-Saturn & $2.85639^{\star} 10^{-4}$ & $65.276555555^{\star} 10^{-12}$ & 00.0010 .01 & $\begin{array}{l}1.00011901 \\
0.99978561 \\
0.99677490\end{array}$ & $\begin{array}{l}0.86602543 \\
0.86602543 \\
0.86602543\end{array}$ \\
\hline Sun-Uranus & $4.36431^{\star} 10^{-5}$ & $6713.99789137^{\star} 10^{-16}$ & 00.0010 .01 & $\begin{array}{l}1.00001818 \\
0.99968474 \\
0.99667373\end{array}$ & $\begin{array}{l}0.86602540 \\
0.86602540 \\
0.86602540\end{array}$ \\
\hline $\begin{array}{l}\text { Sun- } \\
\text { Neptune }\end{array}$ & $5.14805^{\star} 10^{-5}$ & $2037.88920652^{\star} 10^{-16}$ & 00.0010 .01 & $\begin{array}{l}1.00002144 \\
0.99968801 \\
0.99667701\end{array}$ & $\begin{array}{l}0.86602540 \\
0.86602540 \\
0.86602540\end{array}$ \\
\hline
\end{tabular}




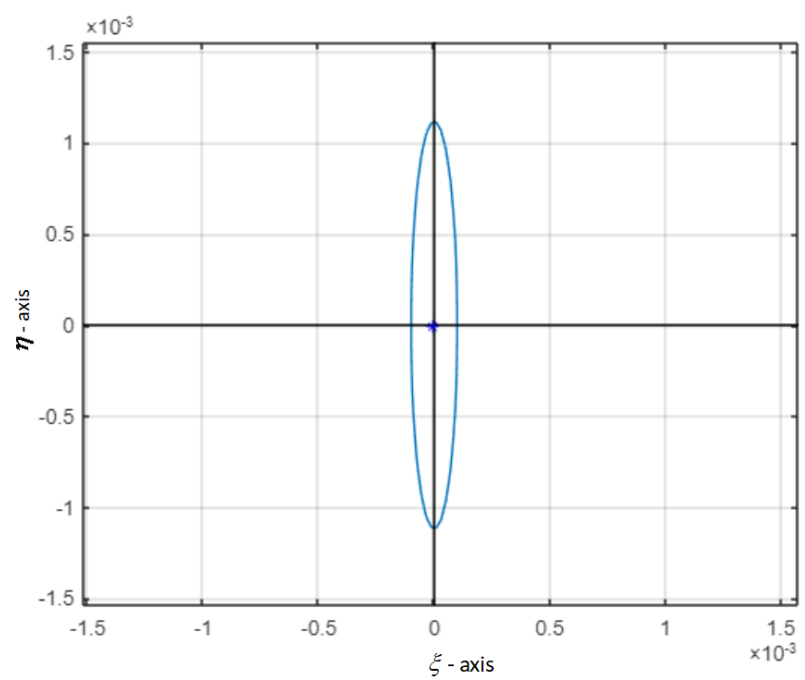

Fig 1. Periodic orbit around the Sun in Sun-Jupiter system for $\left(\mathrm{L}_{1}\right)$ where, semi-major axis $=0.0011881893731$ and semi-major axis $=1 \times 10^{-4}$

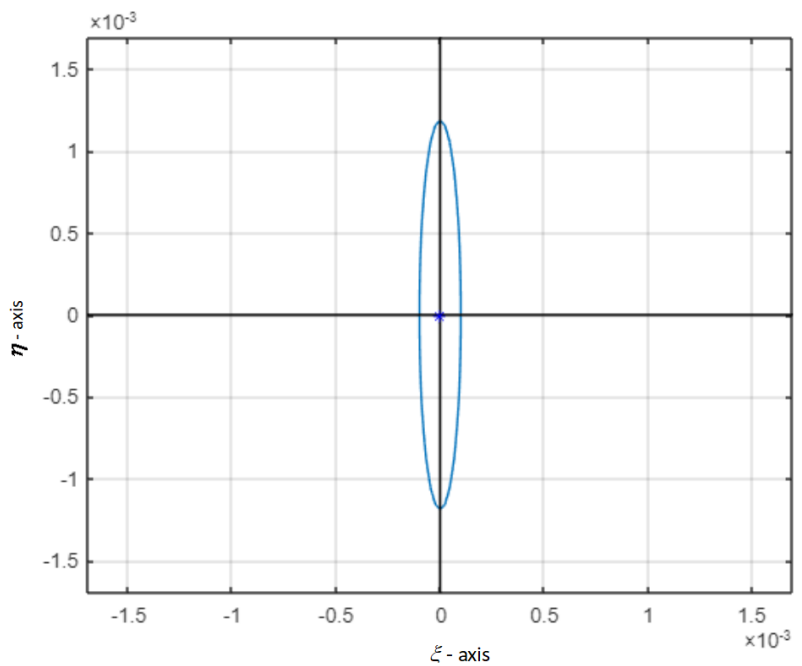

Fig 2. Periodic orbit around the Sun in Sun-Jupiter system for $\left(\mathrm{L}_{1}\right)$ where, semi-major axis $=0.0011881893731$ and semi-major axis $=1 \times 10^{-4}$

\subsection{Stability of the triangular points}

At the triangular points $L_{4}$ and $L_{5}$, we have

$$
\Omega_{x x}=f \text {, }
$$$$
\Omega_{x y}= \pm y[f(x-\mu)+g(x+1-\mu)] \text {, }
$$$$
\Omega_{y y}=y^{2}(f+g)>0 \text {, }
$$

where

$f=\frac{3 q(1-\mu)}{r_{1}^{5}}=\frac{3(1-\mu) n^{2}}{r_{1}^{2}}>0, g=3 \mu\left(1+\frac{5}{2} A_{2}\right)>0$

The characteristics Equation (10) becomes

$$
\Lambda^{2}+\left(4 n^{2}-f r_{1}^{2}-g r_{2}^{2}\right) \Lambda+y^{2} f g=0
$$

with $\Lambda=\lambda^{2}$,

$$
\Lambda_{1,2}=\frac{1}{2}\left(\left(3 \mu A_{2}-n^{2}\right) \pm\left(\left(n^{2}-3 \mu A_{2}\right)^{2}-36 \mu(1-\mu) n^{2}\left(1+\frac{5}{2} A_{2}\right) \times\left(1-\frac{1}{4}\right)\right)^{\frac{1}{2}}\right)
$$

We observe that the roots 


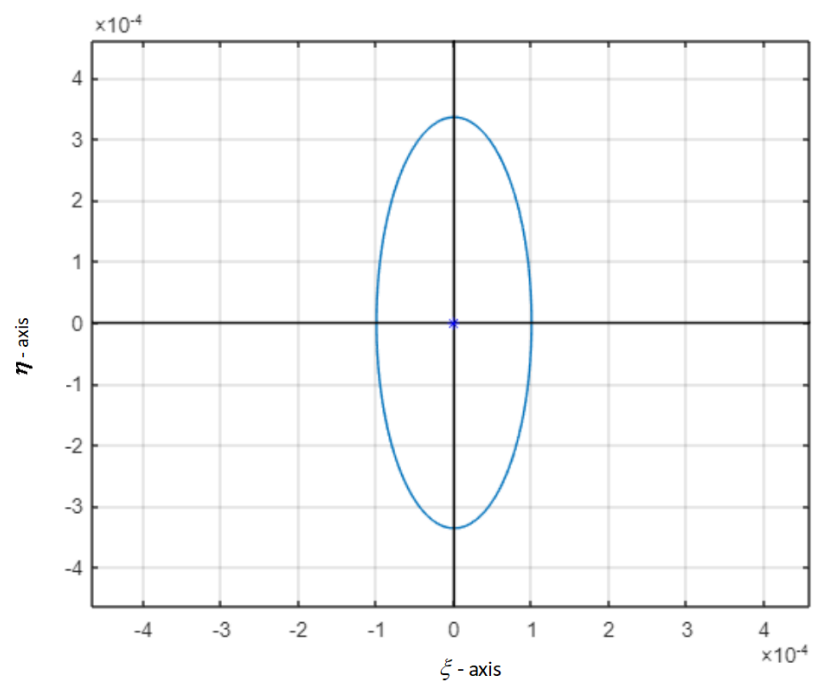

Fig 3. Periodic orbit around the Sun in Sun-Jupiter system for $\left(\mathrm{L}_{2}\right)$ where, semi-major axis $=3.3600985537 \times 10^{-4}$ and semi-major axis $=1 \times 10^{-4}$

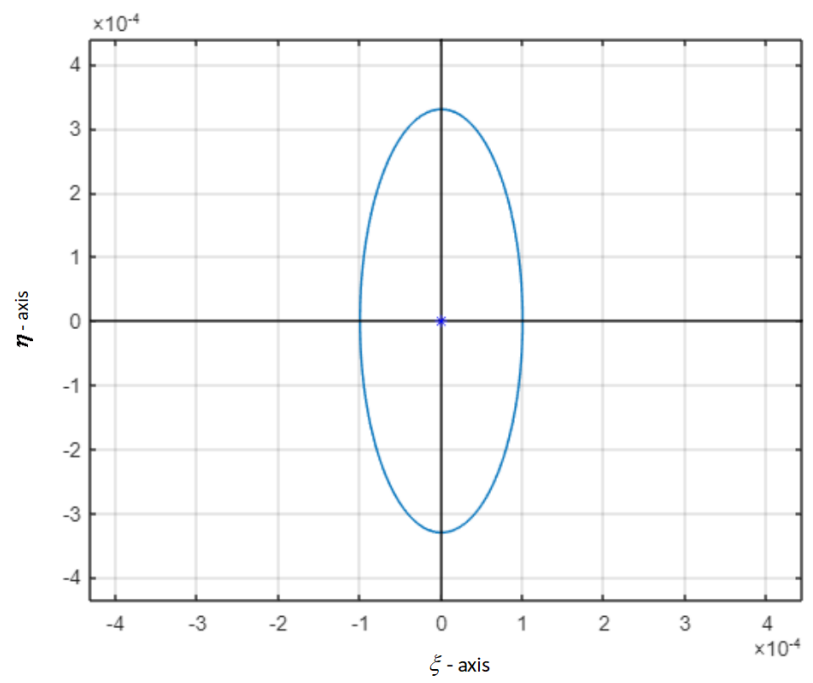

Fig 4. Periodic orbit around the Sun in Sun-Jupiter system for $\left(\mathrm{L}_{2}\right)$ where, semi-major axis $=3.3019851583 \times 10^{-4}$ and semi-major axis $=1 \times 10^{-4}$

$\lambda_{1}=\Lambda_{1}^{\frac{1}{2}}, \lambda_{2}=-\Lambda_{1}^{\frac{1}{2}}, \lambda_{3}=\Lambda_{2}^{\frac{1}{2}}, \lambda_{4}=-\Lambda_{2}^{\frac{1}{2}}(13)$

are functions of $\mu, q$ and $A_{2}$ and their nature depends upon the nature of the discriminant (D).

$D=\left(n^{2}-3 \mu A_{2}\right)^{2}-36 \mu(1-\mu) n^{2}\left(1+\frac{5}{2} A_{2}\right)\left(1-\frac{r_{1}^{2}}{4}\right)$,

And three cases can be discussed as,

When $\mathrm{D}$ is positive, we note that $\Lambda_{1,2}$ are negative and roots (13), written as

$\lambda_{1,2}= \pm i\left(-\Lambda_{1}\right)^{\frac{1}{2}}= \pm i s_{4}, \lambda_{3,4}= \pm i\left(-\Lambda_{2}\right)^{\frac{1}{2}}= \pm i s_{5}$,

Shows the triangular points to be linearly stable.

The solution of Equation (9) in this case can be easily seen to consist of short - and long - period terms with angular frequencies $s_{5}$ and $s_{4}$ respectively. As in ${ }^{(5,8,9)}$ the short-or-long-period terms can be eliminated from the solution with proper selection of initial conditions. In both cases, the motion is along a retrograde ellipse whose eccentricity and the orientation of the major axis are independent of the initial conditions.

When D is negative, the real parts of two of the four roots equation (13) are positive and equal and, hence the equilibria are unstable. However, with suitable selection of initial conditions, periodic motion can be achieved in the linear sense which 


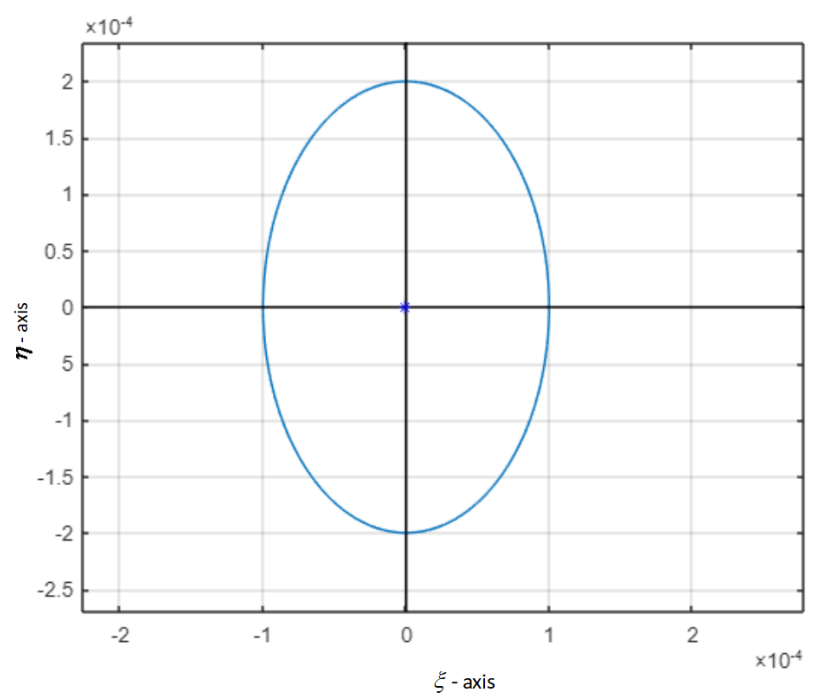

Fig 5. Periodic orbit around the Sun in Sun-Jupiter system for $\left(\mathrm{L}_{3}\right)$ where, semi-major axis $=2.00000208265 \times 10^{-4}$ and semi-major axis $=$ $1 \times 10^{-4}$

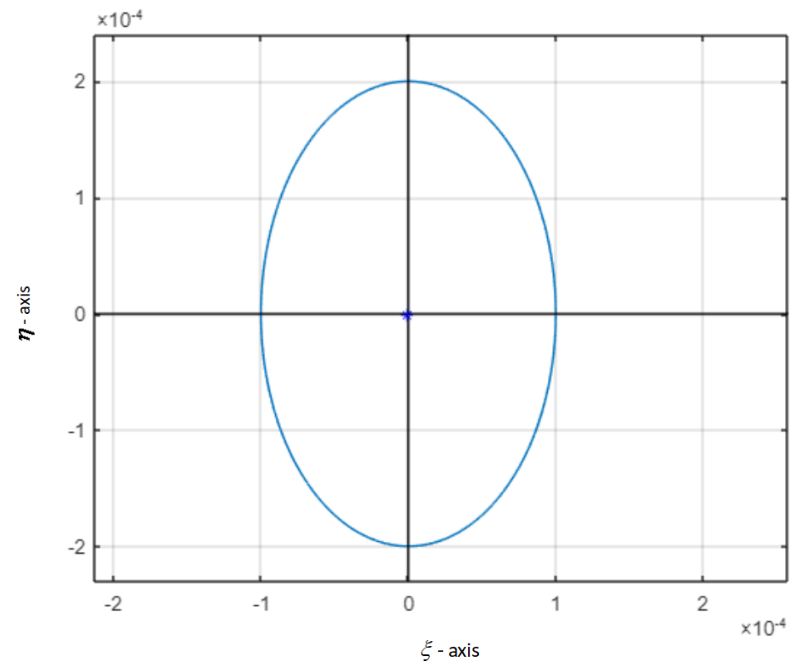

Fig 6. Periodic orbit around the Sun in Sun-Jupiter system for $\left(\mathrm{L}_{3}\right)$ where, semi-major axis $=2.00000209358 \times 10^{-4}$ and semi-major axis $=$ $1 \times 10^{-4}$

approaches the equilibrium point asymptotically.

\section{Critical Mass}

The discriminant of the quadratic Equation (12) is zero when

$$
\left(\frac{-279 A_{2} \varepsilon^{2}}{2}+\varepsilon^{2}+27 A_{2} \varepsilon+6 \varepsilon+\frac{531 A_{2}}{2}+27\right) \mu^{2}+\left(\frac{-279 A_{2} \varepsilon^{2}}{2}+\varepsilon^{2}+27 A_{2} \varepsilon+6 \varepsilon+\frac{489 A_{2}}{2}+27\right) \mu+12 A_{2}+1=0
$$

When $A_{2}=0$, Equation (14) exactly coincides with that of ${ }^{(14)}$.

Solution of the Equation (14) for $0 \leq \mu \leq \frac{1}{2}$ is

$\mu_{\text {crit }}=\frac{-\alpha-\beta^{\frac{1}{2}}}{\gamma},(15)$

Where 


$$
\begin{aligned}
& \alpha=\left(-27-6 \varepsilon-\varepsilon^{2}\right)+\left(\frac{-489}{2}-27 \varepsilon+\frac{279 \varepsilon^{2}}{2}\right) A_{2} \\
& \beta=\left(\sqrt{621}+\frac{50 \sqrt{621} \varepsilon}{207}+\frac{1717 \sqrt{621} \varepsilon^{2}}{42849}\right)+\left(\frac{1205 \sqrt{621}}{138}+\frac{15829 \sqrt{621} \varepsilon}{14283}+\frac{546905 \sqrt{621} \varepsilon^{2}}{85698}\right) A_{2} \\
& \gamma=\left(54+12 \varepsilon+2 \varepsilon^{2}\right)+\left(531+54 \varepsilon-279 \varepsilon^{2}\right) A_{2} \\
& \text { And } \mu_{\text {crit }} \text { expression in }(15) \text { becomes } \\
& \mu_{\text {crit }}=\left(\frac{1}{2}-\frac{\sqrt{621}}{54}\right)-\frac{2 \sqrt{621} \varepsilon}{5589}+\frac{\sqrt{621} \varepsilon^{2}}{42849}+A_{2}\left(\left(\frac{38 \sqrt{621}}{1863}-\frac{7}{18}\right)+\left(\frac{7}{81}-\frac{388 \sqrt{621}}{128547}\right) \varepsilon+\left(\frac{1055}{1458}-\frac{10255 \sqrt{621}}{2313846}\right) \varepsilon^{2}\right] \\
& \text { or, } \\
& \qquad \mu_{\text {crit }}=0.0385209-0.0089174 \varepsilon+0.0005816 \varepsilon^{2}+A_{2}\left(0.119406935+0.011202827 \varepsilon+0.6131487216 \varepsilon^{2}\right)
\end{aligned}
$$

\section{Result}

The value of the Critical mass that we got from the Mean motion $\left(n^{2}\right)=1+6 A_{2}$ is

$\mu_{\text {crit }}=0.0385209-0.0089174 \varepsilon+0.0005816 \varepsilon^{2}+A_{2}\left(0.119406935+0.011202827 \varepsilon+0.6131487216 \varepsilon^{2}\right)$

And the value of the Critical mass that we got from the mean motion expression given by $\left(n^{2}\right)=1+3 / 2 A_{2}{ }^{(3)}$ is

$\mu_{\text {crit }}=0.0385209-0.0089174 \varepsilon+0.0005816 \varepsilon^{2}-A_{2}\left(0.0627795-0.0292011 \varepsilon+0.003436104 \varepsilon^{2}\right)$

First value of oblateness in $\left(\mu_{c r i t}\right)$ or Critical mass is been found out for different values of mean motions $\left(\mathrm{n}^{2}\right)$.

Table 7. $\left(\mu_{\text {crit }}\right)$ or Critical mass parameter is been found out for different values of meanmotions $\left(\mathrm{n}^{2}\right)$.

\begin{tabular}{ll}
\hline$n^{2}$ & $\mu_{\text {crit }}$ (only first term of $\left.\mathbf{A}\right)$ \\
\hline $1+\frac{3}{2} A_{2}$ & -0.06277956 \\
$1+3 A_{2}$ & -0.0020507319 \\
$1+4 A_{2}$ & 0.038435157 \\
$1+5 A_{2}$ & 0.07892104618 \\
$1+6 A_{2}$ & 0.119406935 \\
\hline
\end{tabular}

Graph explaining the mean motion and first value of oblateness in critical mass.

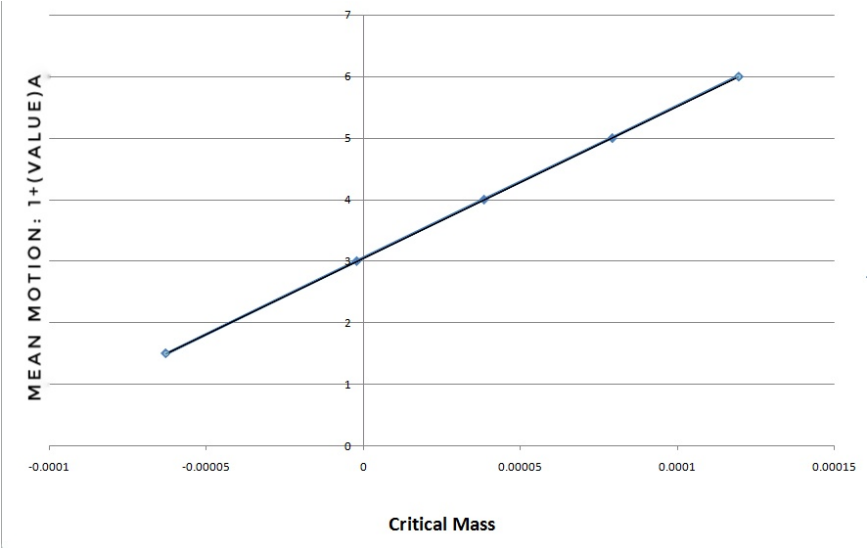

Fig 7. Graph showing the mean motion vs. first value of Oblateness in Critical mass parameter.

\section{Conclusion}

In earlier case of mean motion ' $n$ ' given by $\left(n^{2}\right)=1+3 / 2 A_{2}$ from ${ }^{(3)}$, only secular effect of oblateness on the mean motion was considered $^{(3)}$. However, when the secular effect of the oblateness on the mean motion is considered, argument of perigee and right ascension of the ascending node ${ }^{(1,2)}$, the resulting mean motion ' $n$ ' of the primaries is given by $n^{2}=1+6 \mathrm{~A}_{2}$ is included in the present studies.

In this study, mean motion is increasing since the effect of oblateness on mean motion, argument of perigee and right ascension of ascending node have been considered. All these three parameters are included to get the mean motion of primaries 
$\mathrm{n}^{2}=1+6 \mathrm{~A}_{2}$. The value obtained of the critical mass $\mu$ crit higher than the unperturbed value of $\mu \mathrm{c}=0.0385209$ in $^{(3)}$ with oblateness of the smaller primary. It is a very interesting result, because the zone of mass parameter $\mu$ providing stable solutions at the triangular points increases with oblateness. It can be observed from [ Figure 7 ] and [ Table 7 ] that the increase in oblateness increases the mean motion $\mathrm{n}$ and increases the value of critical mass parameter $\mu$ crit.

\section{References}

1) Johnson A, Sharma RK. Locations of Lagrangian points and periodic orbits around triangular points in the photo gravitational elliptic restricted three-body problem with oblateness. International Journal of Advanced Astronomy. 2019;7(2):25-38. doi:10.14419/ijaa.v7i2.29377.

2) Jency AA, Sharma RK. Location and stability of the triangular Lagrange points in photo-gravitational elliptic restricted three body problem with the more massive primary as an oblate spheroid. International Journal of Advanced Astronomy. 2019;7(2):57-62. doi:10.14419/ijaa.v7i2.29814.

3) Sharma RK. The linear stability of libration points of the photogravitational restricted three-body problem when the smaller primary is an oblate spheroid. Astrophysics and Space Science. 1987;135(2):271-281. doi:10.1007/bf00641562.

4) Rao PVS, Sharma RK. A note on the stability of the triangular points of equilibrium in the restricted three-body problem. Astronomy and Astrophysics. 1975;43:381-383. Available from: http://adsabs.harvard.edu/abs/1975A\%26A....43..381S.

5) Mittal A, Ahmad I, Bhatnagar KB. Periodic orbits in the photogravitational restricted problem with the smaller primary an oblate body. Astrophysics and Space Science. 2009;323(1):65-73. doi:10.1007/s10509-009-0038-2.

6) Patak VON, Thomas EI, Abouelmagd. The perturbed photogravitational restricted three-body problem: Analysis of resonant periodic orbits. Discrete \& Continuous Dynamic Systems-S. American Institute of Mathematical Sciences. 2019;12(4 \& 5):849-875. doi:10.3934/dcdss.2019057.

7) Bello N, Singh J. On the Stability ofL4,5in the Relativistic R3BP with Oblate Secondary and Radiating Primary. Advances in Astronomy. 2015;2015:1-12. doi:10.1155/2015/489120.

8) Pushparaj N, Sharma RK. Interior resonance periodic orbits around the Sun in the Sun-Jupiter photogravitational restricted three-body problem using the method of Poincaré surface of section. Advances in Astrophysics. 2017;2(1):25-34.

9) Das MK, Narang P, Mahajan S, Yuasa M. Effect of radiation on the stability of equilibrium points in the binary stellar systems: RW-Monocerotis, Krüger 60. Astrophysics and Space Science. 2008;314(4):261-274. doi:10.1007/s10509-008-9765-z.

10) Singh J, Amuda TO. Effects of Poynting-Robertson (P-R) drag, radiation, and oblateness on motion around the L4,5 equilibrium points in the CR3BP. Journal of Dynamical Systems and Geometric Theories. 2017;15(2):177-200. doi:10.1080/1726037x.2017.1411043.

11) Szebehely V, and. Theory of Orbits. New York. Academic Press. 1967a;p. 19670055303-19670055303.

12) Danby JMA. Fundamentals of Celestial Mechanics. Willmann-Bell, Inc. 1988.

13) Curtis HD. Orbital Mechanics for Engineering Students. Elsevier. 2014.

14) Chernikov YA. The photogravitational restricted three-body problem. AstronamZ. 1970;47:217-223. 